\title{
Strength, Power and Resistance Training in Older Women
}

\author{
Celia Cohen Barros*, Célia Pereira Caldas \\ Medical Sciences Graduate Program, Medical Sciences Faculty, Rio de Janeiro State University, Rio de Janeiro, \\ Brazil \\ Email: *celiacohen@uol.com.br
}

Received 18 March 2016; accepted 19 April 2016; published 21 April 2016

Copyright (C) 2016 by authors and Scientific Research Publishing Inc.

This work is licensed under the Creative Commons Attribution International License (CC BY). http://creativecommons.org/licenses/by/4.0/

(c) $\underset{\mathrm{EY}}{\mathrm{O}}$ Open Access

\section{Abstract}

Purpose: It has been established that physical exercise generally improves the physical performance of older people. This finding has led to an increase in studies into the most efficient forms of exercise. The aim of this study was to compare the efficiency of three types of exercise: aerobic, strength and muscle power, in improving the functional capacity of older people. Methods: A study was undertaken of 123 physically independent older women who did no physical exercise. The women were placed randomly into four groups: a Control Group (CG, $n=33$ ) who did no exercise; an Aerobic Group (AG, $n=30$ ) who did mild intensity muscle resistance exercises, combined with medium intensity gait training; a Strength Group (SG, $n=30$ ) which performed high resistance localized exercises; and a Power Group (PG, $n=30$ ) which did high-speed localized medium resistance exercises. The tests applied were aerobic endurance: six minute walking test (6MWT); maximum strength: one repetition maximum test (1RM); power: Tendo Weightlifting Analyzer (TWA); and agility: standing and walking. AG, SG and PG underwent 24 exercise sessions. Results: All the groups except for CG demonstrated significant improvement in all the tests applied. There were non-statistically significant differences between the gains made by the AG, SG and PG groups in the 6MWT, power and agility tests. SG was significantly superior to the other groups in the 1RM test. Conclusions: Aerobic, strength and muscle training improved the functional capacity of older people. SG was notably superior to the other groups in the 1RM test.

\section{Keywords}

Older People, Aging, Physical Exercise, Health

\section{Introduction}

All physical abilities tend to decline as a result of aging. Aerobic endurance, muscle strength and muscle power *Corresponding author. 
(strength $\mathrm{x}$ velocity) are, among other factors, potential elements to affect the cardio-circulatory, respiratory and muscle systems, which are essential for maintaining the health and capacity to perform activities of daily living.

The process of decline can be reduced by physical exercise [1]. Maintaining and improving physiological capacities through aerobic, strength or power training programs result in beneficial outcomes, as they minimize the effects of the aging process, demonstrating that older people maintain their ability to adapt to physical exercise.

The purpose of this study was to evaluate and compare the effectiveness of three kinds of training programs targeted for improving strength, power, aerobic endurance and agility.

\section{Methods}

A total of 123 older female volunteers were included according with the following criteria: $\geq 60$ years old; physically independent; not attending any physical exercise program and; able to perform all the proposed exercises and tests. Individuals suffering from joint or muscle diseases, physical or mental disability, or heart disease were excluded.

The population was randomly stratified into four groups: an aerobic training group (AG $\mathrm{n}=30$ ); a strength training group ( $\mathrm{SG} n=30)$; a power training group $(\mathrm{PG} n=30)$ and a Control Group $(\mathrm{CG} n=33)$.

After a medical evaluation to verify their health status, the able participants attended six sessions to familiarize with the tests and exercises. These sessions were aimed to teach the movements of the exercises and the testing procedures. The same training exercises and testing were used, but without load.

Muscle power (watts-W) was measured by means of a power curve for the knee extensor exercise using a leg extension machine, a method validated by Jennings et al. [2]. The computerized Tendo Weightlifting AnalyzerModel V104 (Tendo, Slovakia) was used to identify and monitor strength and power training.

Maximum strength (kilograms-kg) was measured by the one repetition maximum test (1RM) of the knee extensor exercise, performed with a leg extension machine (Righetto Fitness Equipment, Brazil), following the procedures recommended by the American College of Sports Medicine (ACSM) [3]. The aerobic capacity (meters-m) was measured by the six-minute walk test (6MWT), validated by Rikli and Jones [4]. Agility was measured by the standing and walking test. This test also verifies dynamic balance.

\section{Intervention}

The parameters used in the intervention followed ACSM [3] and American Heart Association (AHA) [5] recommendations as well as those of the US Department of Health and Human Services (HHS) [6].

Knee extensor, knee flexion, leg press, hip adduction, biceps and triceps curl, and lateral elevation of the upper limbs exercises were used for training the AG, SG and PG groups, although the intensity and form of execution varied. For AG exercise sessions consisted of 20 minutes of moderate intensity gait training and two sets of 15 repetitions of exercises at an intensity of 30\% 1RM. For SG three sets of eight repetitions at an intensity of $80 \% 1 \mathrm{RM}$ were performed. For PG exercises were performed in three sets of eight repetitions at maximum speed during the concentric phase at an intensity of $80 \%$ of maximum strength, registered with the highest average power for the equipment Tendo Weightlifting Analyzer.

Training frequency for all groups was three times per week on alternate days for 24 total sessions. The control group underwent the same evaluation methodology as the intervention groups. They were instructed to maintain their normal daily activities and advised not to start any kind of training that involved strength exercises with weights or aerobic activities.

All tests performed as part of the pre-intervention procedures were repeated during the post-intervention phase.

\section{Statistical Analysis}

Descriptive analysis and statistical tests were used to evaluate intragroup, intergroup and pre- and post-intervention differences. The Student's t-test for paired samples was used for intragroup comparison, and ANOVA was used for comparison between groups. The level of significance was $\mathrm{p}<0.05$.

The research was conducted in accordance with the Declaration of Helsinki, and was approved by the Ethics Committee of the State University of Rio de Janeiro. All volunteers completed an informed consent form before participating in this study. 


\section{Results}

The women included were 70 years old on average, with a standard deviation (SD) of 8.5 years (Aerobic Group 71, SD of 5.6; Strength Group 69.4, SD of 4.1; Power Group 71.1, SD of 5.6; Control Group 71, SD of 4.9). The average height was 1.60 meters, with a standard deviation of 0.05 (AG 1.57, SD of 0.03; SG 1.58, SD of 0.01 ; PG 1.57, SD of 0.02; CG 1.58, SD of 0.01). The weight of women was 65.8 on average with a standard deviation of $5.3 \mathrm{~kg}$ (AG $62.9 \mathrm{Kg}$, SD of $5.6 \mathrm{~kg}$; SG 65.3, SD of $1.4 \mathrm{~kg}$; PG 63.4, SD of 4.1 kg; CG 62.7, SD of 3.7 $\mathrm{kg})$.

Regarding race, the Aerobic Group had 76.6\% white women; 13.3\% multiracial and 10\% black women. The Strength Group had 80\% white women; 10\% multiracial and 10\% black women. Power Group had 73.3\% white women; $13.3 \%$ multiracial and $13.3 \%$ black women. Control Group had 78.7\% white women; $12.5 \%$ multiracial and $9 \%$ black women.

\subsection{Intragroup Results}

Table 1 shows the results obtained in the 1RM, 6MWT, muscle power and agility tests. It can be seen that training groups (AG, SG and $\mathrm{PG}$ ) showed significant improvement in all tests in comparison with the period prior to exercise training, while the control group showed no significant improvement.

\subsection{Intergroup Results}

\subsubsection{Aerobic Endurance Test}

The results of aerobic endurance test (6MWT) are shown in Figure 1. The CG had the lowest average endurance gain $(\mathrm{p}<0.001)$, while the differences between other groups $(\mathrm{AG}, \mathrm{SG}$ and $\mathrm{PG})$ were not statistically significant. AG showed greatest gain in the 6MWT test, followed by PG and SG (Figure 1(a)).

\subsubsection{Muscle Strength Test}

Figure 1(b) shows results of muscle strength test (1RM). The PG showed greater gain in muscle strength than the other groups, while CG had the lowest rate. The difference between these groups was statistically significant $(\mathrm{p}<0.001)$. The difference between gains of AG and PG was not significant (Figure 1(b)).

Table 1. Results of 1RM, 6MWT, muscle power and agility tests for groups studied.

\begin{tabular}{|c|c|c|c|c|c|}
\hline Tests & Groups & Pre-intervention & Post-intervenion & Gain & P Value \\
\hline \multirow{4}{*}{$\begin{array}{l}1 \mathrm{RM} \\
(\mathrm{kg})\end{array}$} & CG & $13.72 \pm 4.49$ & $13.60 \pm 4.73$ & -0.12 & 0.62 \\
\hline & AG & $12.05 \pm 3.6$ & $16.45 \pm 3.8$ & 4.40 & $<0.001$ \\
\hline & SG & $14.90 \pm 4.09$ & $21.03 \pm 5.10$ & 6.13 & $<0.001$ \\
\hline & PG & $14.83 \pm 3.96$ & $19.93 \pm 4.21$ & 5.10 & $<0.001$ \\
\hline \multirow{4}{*}{$\begin{array}{c}\text { 6MWT } \\
(\mathrm{m})\end{array}$} & $\mathrm{CG}$ & $503 \pm 65.12$ & $509 \pm 54.73$ & 6.00 & 0.16 \\
\hline & AG & $500 \pm 64.00$ & $536 \pm 56.40$ & 36.00 & $<0.001$ \\
\hline & SG & $536 \pm 72.49$ & $561 \pm 69.46$ & 25.00 & $<0.001$ \\
\hline & PG & $522 \pm 48.83$ & $549 \pm 56.62$ & 27.00 & $<0.001$ \\
\hline \multirow{4}{*}{$\begin{array}{l}\text { Muscle Power } \\
\text { (W) }\end{array}$} & $\mathrm{CG}$ & $46.90 \pm 15.68$ & $46.72 \pm 15.72$ & -0.18 & 0.90 \\
\hline & $\mathrm{AG}$ & $43.83 \pm 13.64$ & $56.23 \pm 17.36$ & 12.40 & $<0.001$ \\
\hline & SG & $52.50 \pm 19.30$ & $67.50 \pm 17.12$ & 15.00 & $<0.001$ \\
\hline & PG & $51.40 \pm 13.03$ & $63.90 \pm 15.09$ & 12.50 & $<0.001$ \\
\hline \multirow{4}{*}{$\begin{array}{l}\text { Agility } \\
\text { (s) }\end{array}$} & $\mathrm{CG}$ & $5.60 \pm 0.99$ & $5.74 \pm 0.99$ & 0.14 & 0.13 \\
\hline & $\mathrm{AG}$ & $5.62 \pm 0.66$ & $5.15 \pm 0.62$ & -0.47 & $<0.001$ \\
\hline & SG & $5.09 \pm 1.10$ & $4.74 \pm 0.99$ & -0.35 & $<0.001$ \\
\hline & PG & $5.54 \pm 0.86$ & $5.16 \pm 0.75$ & -0.38 & $<0.001$ \\
\hline
\end{tabular}




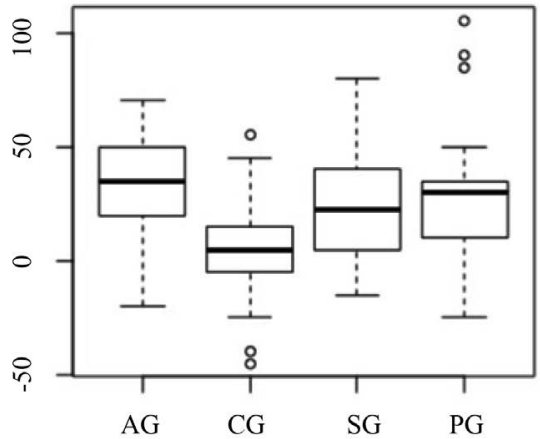

(a)

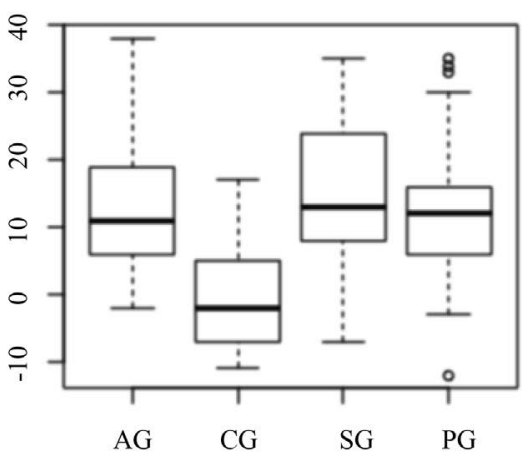

(c)

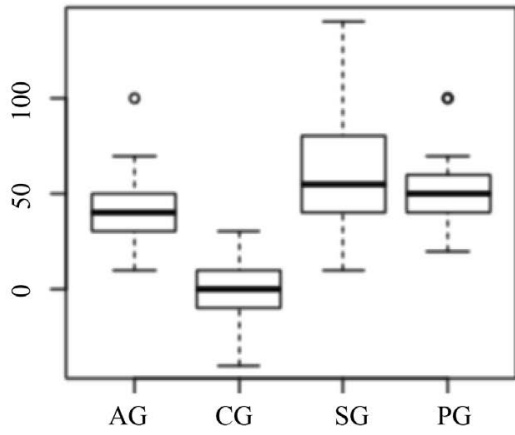

(b)

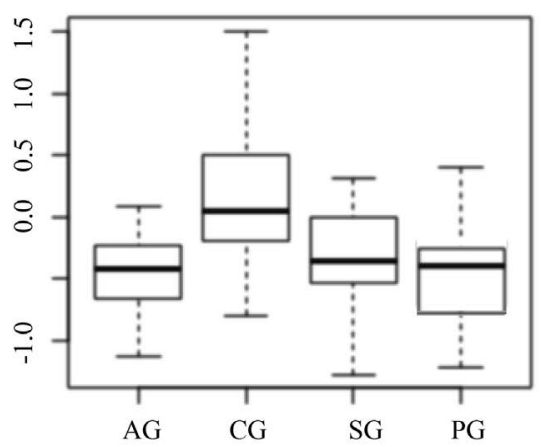

(d)

Figure 1. (a) Mean obtained in endurance test (6MWT) in meters (m) for each group; (b) Mean obtained in strength test (1RM) in kilograms (kg) for each group; (c) Mean obtained in muscle power test, in watts (W) for each group; (d) Mean obtained in agility test, in seconds, for each group.

\subsubsection{Muscle Power Test}

The results of muscle power test are shown in Figure 1(c). The CG had the lowest improvement, and this difference was statistically significant $(\mathrm{p}<0.001)$. The SG had the biggest gain, followed by PG and AG (Figure $1(c))$.

\subsubsection{Agility Test}

The results of agility test are shown in Figure 1(d). The CG had significantly higher mean result than the other groups, signifying at the least, improvement $(\mathrm{p}<0.001)$. The difference between the other groups was not statistically significant, although AG had the biggest gain, followed by PG and SG (Figure 1(d)).

\section{Discussion}

It is a known fact that exercise produces beneficial effect on older person's physical performance [1] [7]-[9]. However, it was expected that there would be significant differences between the different training groups.

While the richness and complexity of the aging process explain some results that did not confirm this initial hypotheses, the results obtained confirmed the benefits of systematic practice of physical exercise [1] [7]-[9].

The three training groups showed improvement for all tests, while the Control Group did not show any benefit and its initial levels of physical performance remained unchanged.

The aerobic exercises performed by AG increased strength. The localized muscle resistance exercise included in the training protocol for this group, may have contributed to this result. However it should be pointed out that aerobic training also increased strength. Similar results were described by Harber et al. [7] and Ozaki et al. [10].

As expected, the high intensity strength exercises performed by SG increased strength, which shows the adaptability of muscle tissue in older persons in response to intensive effort. McArdle et al. [11] and Häkkinen et al. [12] report that increased strength is primarily caused by neuromuscular factors; while Melov et al. [13] 
and Raymond et al. [14] claim that there is an increase in the size and number of muscle fibers.

The muscle power exercises applied by the PG group also proved effective in increasing the muscle strength. As power is a derivative of strength which, in turn, is associated with speed, the result was consistent with the findings of studies into the effectiveness of muscle power exercises at increasing the strength of older people [1] [15] [16].

The gain in distance walked indicates that aerobic training significantly increased aerobic endurance. This improvement was particularly noticeable during exercise sessions in which the adaptability to increase in requested stimuli was observed. Although, no hemodynamic and metabolic variables were measured, the results of this study help to reinforce the benefits of aerobic exercise [1]. The increases in distance walked by the SG and PG also indicate that high intensity strength exercises and muscle power exercise significantly increased aerobic endurance.

In addition to strength and power there is a secondary increase in muscle resistance. It is believed that the main factor behind this increase is an improvement in motor efficiency, which occurs through reduced activation of the motor units [16] and antagonistic muscle activity [17], increased availability of high-energy phosphate [18], and greater mitochondrial density and oxidative capacity [19]. Significant improvements in muscle strength have been reported after moderate and strong intensity strength training [20]-[22]. The present study reinforces the findings of mentioned literature.

The muscle power production capacity gain indicates that aerobic training significantly increased the muscle power production capacity of subjects. As with the strength gain results, it is likely that the localized muscle strength exercise included in aerobic training contributed to this result. As power is related to strength, the result shows a consistent correlation between the two components.

The SG group increased average muscle power production capacity by $15 \mathrm{~W}$, with a margin of error of $<0.001$, indicating that high intensity muscle strength training can significantly increase muscle power capacity. Studies featuring different resistances and intensities obtained similar responses [23] [24].

Muscle power capacity increased by a mean of $12.50 \mathrm{~W}$ in the PG group, with a margin of error of $<0.001$, indicating that muscle power training significantly increased the muscle power production capacity of subjects. Similar results were obtained in previous studies using the same methodology, confirming the effectiveness of this training method in increasing muscle power production capacity among older people [25].

The results of agility test showed that aerobic, strength and muscle power training were able to significantly increase the agility of the groups studied.

When results for each test were compared between groups, it was found that CG was the only group with significantly lower results than the other groups in all tests, confirming that those who do not exercise and do not improve their physical performance. As reported, the AG, SG and PG groups achieved significant gains in all the tests. There was a difference between the mean results of groups for each test, but the difference was not significant for endurance testing (6MWT), power and agility. For strength test (1RM), SG had achieved significantly higher mean results than the other groups.

Although there was some variation between the mean aerobic testing results, with AG showing greater improvement, followed by PG and SG, respectively, the differences between the groups were not significant.

The expected hypothesis that the AG group would show a significantly greater improvement than the SG and PG groups in the endurance test, given the specificity of this training method to the test, did not occur. It should be noted that the 6MWT is a field test that correlates well with maximum laboratory tests [4]. The positive results presented by SG and PG may be related to the fact that the 6MWT is a sub-maximal test, as the ability to adapt to strength and muscle power training can result in adaptation to sub-maximal aerobic effort as a secondary benefit, as some studies have shown [20]-[22].

In strength test SG showed significantly higher mean results. Even though PG displayed greater gain in the strength test than AG, the difference was not significant. However, high intensity strength training was more effective for increasing maximum strength than the other types of training tested in the study. The response of groups to the 1RM test corresponded with the expected hypothesis. It is likely that the training intensity factor may have contributed to the result, as literature reports that high intensity strength training is more effective for gaining maximum strength.

Importantly, even with different types of exercise or exercises performed at different intensities, muscle strength and aerobic training were able to significantly improve the strength gain of the groups, according to some studies [10] [26]. 
Even though the mean results of the power test varied, with the SG group showing the greatest improvement, followed by PG and AG, respectively, the differences were not significant. There was an expectation that PG would have a significantly higher mean than the other groups in the power test, due to its specific nature, but this did not occur. Some studies [27] [28] have found that muscle strength and power increased in accordance with the intensity of the exercises. In the present study, the three groups exercised at different intensities, but there were no significant differences between groups. The SG, which underwent strength training, had the best results. Considering that $\mathrm{P}=\mathrm{FxV}$ (where $\mathrm{P}=$ power, $\mathrm{F}=$ force and $\mathrm{V}=$ velocity), and that these factors are closely related, while the amount of power generated depends on the mass to be dislocated, power also depends on the speed at which displacement occurs. It follows from this that the larger the mass, the lower the possibility of the movement developing speed, if forces of the same magnitude are considered. This is why the greatest power generation is obtained from an optimal relationship between the magnitude of the displaced mass and movement speed.

In a previous study into the effectiveness of power training among older people, Barros et al. [25] observed that an increase in the ability to generate muscle power occurred more because of greater displacement of resistance than because of increased movement speed. In this case the gain in strength led to improved muscle power. In the present study, the SG group had significantly greater strength gains than the other groups, which probably contributed to SG having better results than PG for the power test, even if these results were not significant.

While the mean results were different, with the AG group showing the greatest mean gain in the agility test, followed by PG and SG, respectively, the difference was not significant. Literature reveals improvements in the performance of daily motor tasks resulting from different types of training. Some authors have tried to identify the most specific methods for this purpose in studies by comparing different types, intensities or combinations of exercise [19] [26] [29].

In a comparison of the types of exercise in the present study, aerobic exercise had the highest mean score for medium intensity walking combined with low intensity muscle resistance exercises, followed by power training, and lastly strength training. Literature reports that combined exercises (strength and endurance) and power training are more related to the improvement of motor tasks among older people than strength exercises [1]. In the present study, the same trend was identified, although the differences were not significant.

\section{Study limitations}

The fact that the present study did not use equipment to standardize movement speed during the PG power training exercises, but instead instructed subject that the exercises should be performed at the maximum possible speed, is a potential limitation.

The intervals between sets of exercises were not uniform, as it is understandable that the older individuals needed different periods to recover from stress.

\section{Conclusions}

The three training programs were proved effective to improve the functional capacity of subjects. There was significant increase in aerobic performance, strength, muscle power and agility. Therefore, it can be inferred that a training program with more than 24 total sessions would probably improve the physical performance more remarkably.

With the exception of the control group, the differences between the results of all intervention groups for the endurance, power and agility tests were not significant.

The results of the strength group were significantly better than the other groups in the 1RM test, indicating that this form of exercise is more effective for muscle strength gain.

\section{Funding}

The funding for this study has been provided by the Higher Education Personnel Improvement Coordination (Capes), foundation of the Brazilian Ministry of Education.

\section{Conflict of Interest}

There is no conflict of interest to be reported. 


\section{References}

[1] Chodzko-Zajko, W.J., Proctor, D.N., Fiatarone-Singh, M.A., et al. (2009) American College of Sports Medicine. American College of Sports Medicine Position Stand. Exercise and Physical Activity for Older Adults Position Stand. Medicine \& Science in Sports \& Exercise, 41, 1510-1530. http://dx.doi.org/10.1249/MSS.0b013e3181a0c95c

[2] Jennings, C.L., Viljoen, W., Durandt, J. and Lambert, M.I. (2005) The Reliability of the Fitro Dyne as a Measure of Muscle Power. The Journal of Strength \& Conditioning Research, 19, 859-863.

[3] Rikli, R.E. and Jones, C.E. (1998) The Reliability and Validity of a 6-Minute Walk Test as a Measure of Physical Endurance in Older Adults. Journal of Aging and Physical Activity, 6, 363-375.

[4] Kramer, W.J., Adams, K., Cafarelli, E., et al. (2002) American College of Sports Medicine. American College of Sports Medicine Position Stand. Progression Models in Resistance Training for Healthy Adults. Medicine \& Science in Sports \& Exercise, 34, 364-380. http://dx.doi.org/10.1097/00005768-200202000-00027

[5] Williams, M.A., Haskell, W.L., Ades, P.A., et al. (2007) American Heart Association Council on Clinical Cardiology; American Heart Association Council on Nutrition, Physical Activity, and Metabolism. Resistance Exercise in Individuals with and without Cardiovascular Disease-2007 Update: A Scientific Statement from the American Heart Association Council on Clinical Cardiology and Council on Nutrition, Physical Activity, and Metabolism. Circulation, 116, 572-584. http://dx.doi.org/10.1161/CIRCULATIONAHA.107.185214

[6] (2009) Physical Activity Guidelines Advisory Committee Report, 2008: To the Secretary of Health and Human Services. Part A: Executive Summary. Nutrition Reviews, 67, 114-120.

[7] Harber, M.P., Konopka, A.R., Undem, M.K., et al. (2012) Aerobic Exercise Training Induces Skeletal Muscle Hypertrophy and Agedependent Adaptations in Myofiber Function in Young and Older Men. Journal of Applied Physiology, 113, 1495-1504. http://dx.doi.org/10.1152/japplphysiol.00786.2012

[8] Hunter, G.R., McCarthy, J.P. and Bamman, M.M. (2004) Effects of Resistance Training on Older Adults. Sports Medicine, 34, 329-348. http://dx.doi.org/10.2165/00007256-200434050-00005

[9] Hunter, G.R., Treuth, M.S., Weinsier, R.L., et al. (1995) The Effects of Strength Conditioning on Older Women's Ability to Perform Daily Tasks. Journal of the American Geriatrics Society, 43, 756-760. http://dx.doi.org/10.1111/j.1532-5415.1995.tb07045.x

[10] Ozaki, H., Loenneke, J.P., Thiebaud, R.S., Stager, J.M. and Abe, T. (2013) Possibility of Leg Muscle Hypertrophy by Ambulation in Older Adults: A Brief Review. Journal of Clinical Interventions in Aging, 8, 369-375.

[11] McArdle, W.D., Katch, F.L. and Katch, V.L. (2011) Exercise Physiology: Energy, Nutrition, and Human Performance. 7th Edition, Guanabara-Koogan, Rio de Janeiro, 518

[12] Häkkinen, K., Alen, M., Kallinen, M., Newton, R.U. and Kraemer, W.J. (2000) Neuromuscular Adaptation during Prolonged Strength Training, Detraining and Re-Strength-Training in Middle-Aged and Elderly People. European Journal of Applied Physiology, 83, 51-62. http://dx.doi.org/10.1007/s004210000248

[13] Melov, S., Tarnopolsky, M.A., Beckman, K., Felkey, K. and Hubbard, A. (2007) Resistance Exercise Reverses Aging in Human Skeletal Muscle. PLoS ONE, 2, e465. http://dx.doi.org/10.1371/journal.pone.0000465

[14] Raymond, M.J., Bramley-Tzerefos, R.E., Jeffs, K.J., Winter, A. and Holland, A.E. (2013) Systematic Review of High-Intensity Progressive Resistance Strength Training of the Lower Limb Compared with Other Intensities of Strength Training in Older Adults. Archives of Physical Medicine and Rehabilitation, 94, 1458-1472. http://dx.doi.org/10.1016/j.apmr.2013.02.022

[15] Izquierdo, M., Häkkinen, K., Ibañez, J., Garrues, M., Antón, A., Zúñiga, A., et al. (2001) Effects of Strength Training on Muscle Power and Serum Hormones in Middle-Aged and Older Men. Journal of Applied Physiology, 90, 14971507.

[16] Newton, R.U., Häkkinen, K., Häkkinen, A., McCormick, M., Volek, J. and Kraemer, W.J. (2002) Mixed-Methods Resistance Training Increases Power and Strength of Young and Older Men. Medicine \& Science in Sports \& Exercise, 34, 1367-1375. http://dx.doi.org/10.1097/00005768-200208000-00020

[17] Häkkinen, K., Newton, R.U., Gordon, S.E., McCormick, M., Volek, J.S., Nindl, B.C., et al. (1998) Changes in Muscle Morphology, Electromyographic Activity, and Force Production Characteristics during Progressive Strength Training in Young and Older Men. The Journals of Gerontology. Series A, Biological Sciences and Medical Sciences, 53, B415B423. http://dx.doi.org/10.1093/gerona/53A.6.B415

[18] Jubrias, S.A., Esselman, P.C., Price, L.B., Cress, M.E. and Conley, K.E. (2001) Large Energetic Adaptations of Elderly Muscle to Resistance and Endurance Training. Journal of Applied Physiology, 90, 1663-1670.

[19] Karavirta, L., Häkkinen, A., Sillanpää, E., García-López, D., Kauhanen, A., Haapasaari, A., et al. (2011) Effects of Combined Endurance and Strength Training on Muscle Strength, Power and Hypertrophy in 40-67-Year-Old Men. Scandinavian Journal of Medicine \& Science in Sports, 21, 402-411. 
http://dx.doi.org/10.1111/j.1600-0838.2009.01059.x

[20] Adams, K.J., Swank, A.M., Berning, J.M., Sevene-Adams, P.G., Barnard, K.L. and Shimp-Bowerman, J. (2001) Progressive Strength Training in Sedentary, Older African American Women. Medicine \& Science in Sports \& Exercise, 33, 1567-1576. http://dx.doi.org/10.1097/00005768-200109000-00021

[21] Grimby, G., Aniansson, A., Hedberg, M., Henning, G.B., Grangård, U. and Kvist, H. (1992) Training Can Improve Muscle Strength and Endurance in 78- to 84-Yr-Old Men. Journal of Applied Physiology, 73, 2517-2523.

[22] Vincent, K.R., Braith, R.W., Feldman, R.A., Magyari, P.M., Cutler, R.B., Persin, S.A., et al. (2002) Resistance Exercise and Physical Performance in Adults Aged 60 to 83. Journal of the American Geriatrics Society, 50, 1100-1107. http://dx.doi.org/10.1046/j.1532-5415.2002.50267.x

[23] Ferri, A., Scaglioni, G., Pousson, M., Capodaglio, P., Van Hoecke, J. and Narici, M.V. (2003) Strength and Power Changes of the Human Plantar Flexors and Knee Extensors in Response to Resistance Training in Old Age. Acta Physiologica Scandinavica, 177, 69-78. http://dx.doi.org/10.1046/j.1365-201X.2003.01050.x

[24] Fielding, R.A., Le Brasseur, N.K., Cuoco, A., Bean, J., Mizer, K. and Fiatarone Singh, M.A. (2002) High-Velocity Resistance Training Increases Skeletal Muscle Peak Power in Older Women. Journal of the American Geriatrics Society, 50, 655-662. http://dx.doi.org/10.1046/j.1532-5415.2002.50159.x

[25] Barros, C.C., Caldas, C.P. and Batista, L.A. (2013) Influence of Muscle Power Training on the Ability to Implement Motor Tasks for Older Women. Revista Brasileira de Geriatria e Gerontologia, 16, 603-613. http://dx.doi.org/10.1590/S1809-98232013000300017

[26] Izquierdo, M., Häkkinen, K., Ibáñez, J., Kraemer, W.J. and Gorostiaga, E.M. (2005) Effects of Combined Resistance and Cardiovascular Training on Strength, Power, Muscle Cross-Sectional Area, and Endurance Markers in Middle-Aged Men. European Journal of Applied Physiology, 94, 70-75. http://dx.doi.org/10.1007/s00421-004-1280-5

[27] Cadore, E.L. and Isquierdo, M. (2013) How to Simultaneously Optimize Muscle Strength, Power, Functional Capacity, and Cardiovascular Gains in the Elderly: An Update. Age, 35, 2329-2344. http://dx.doi.org/10.1007/s11357-012-9503-x

[28] Cadore, E.L., Izquierdo, M., Conceição, M., Radaelli, R., Pinto, R.S., Baroni, B.M., et al. (2012) Echo Intensity Is Associated with Skeletal Muscle Power and Cardiovascular Performance in Elderly Men. Experimental Gerontology, 47, 473-478. http://dx.doi.org/10.1016/j.exger.2012.04.002

[29] Cadore, E.L., Pinto, R.S., Bottaro, M. and Izquierdo, M. (2014) Strength and Endurance Training Prescription in Healthy and Frail Elderly. Aging and Disease, 5, 183-195. http://dx.doi.org/10.14336/AD.2014.0500183 